\title{
Numerical Estimation of the Influence of Yb:YAG Laser Beam Caustic on the Formation of Welding Pool in Laser-Arc Hybrid Welding Process
}

\author{
M. KUBIAK* \\ Częstochowa University of Technology, al. Armii Krajowej 19, 42-201 Częstochowa, Poland \\ Doi: 10.12693/APhysPolA.138.218 \\ *e-mail: kubiak@imipkm.pcz.pI
}

\begin{abstract}
The major aim of this work is the numerical analysis of the influence of Yb:YAG laser beam power distribution in the function of laser beam caustics on the formation of welding pool in butt welded sheets made of S355 steel. The laser beam heat source is modelled on the basis of geostatistical kriging interpolation method using measurements of Yb:YAG laser intensity for different beam focusing. Thermal phenomena with fluid flow in the melted zone are described by equations of mass, momentum, and energy conservation. Differential governing equations are solved using Chorin's projection with finite volume method on staggered grid. Obtained results include temperature field and melted material velocity field. Numerically predicted welding pool geometry in the cross-section of the joint is partially verified by experimental results.
\end{abstract}

topics: hybrid welding, Yb:YAG, thermal phenomena, numerical modelling

\section{Introduction}

Many issues concerning welding processes using $\mathrm{Yb}$ :YAG lasers with the active element in a shape of a disk are currently under particular investigations in the field of mathematical and numerical modelling, as well as experimental research [1]. The main factor characterizing the temperature distribution in the heated element is the amount of heat energy and a method for its delivery to the heated material [2]. Therefore, laser beam focusing has a direct impact on the temperature field and in consequence on the formation of melted zone in welding process using a laser beam.

The aim of this work is the analysis of the influence of Yb:YAG laser beam power distribution in the function of laser beam caustics on the welding pool geometry and size. Three computer simulations of the process are performed for different laser beam focusing. Differential governing equations are numerically solved using projection method with finite volume method (FVM) [3]. The spatial variables are discretized using staggered grid to avoid odd-even decoupling between the pressure and velocity. Scheme of considered system is presented in Fig. 1.

\section{Mathematical and numerical model}

\subsection{Governing equations}

Differential governing equations used for the analysis of thermal phenomena in laser welding process consist of mass, momentum and energy conservation equations:

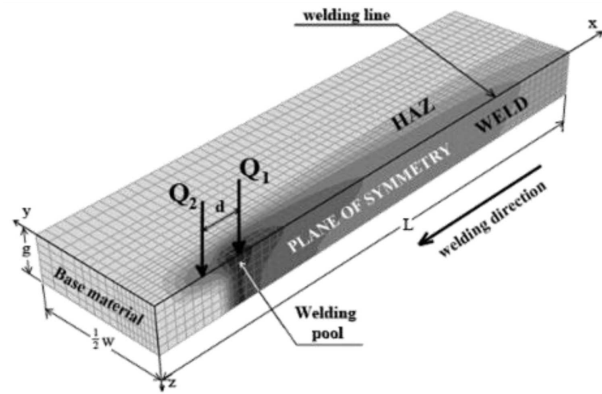

Fig. 1. Scheme of considered system.

$$
\begin{aligned}
& \frac{\partial \rho}{\partial t}+\frac{\partial}{\partial x_{i}}\left(\rho v_{i}\right)=0 \\
& \frac{\partial\left(\rho v_{i}\right)}{\partial t}+\frac{\partial}{\partial x_{j}}\left(\rho v_{i} v_{j}\right)=-\frac{\partial p}{\partial x_{i}}+\frac{\partial}{\partial x_{j}}\left(\mu \frac{\partial v_{i}}{\partial x_{j}}\right) \\
& \quad+\boldsymbol{g} \beta_{T}\left(T-T_{\mathrm{ref}}\right)-\frac{\mu}{\rho K} v_{i} \\
& \frac{\partial}{\partial x_{i}}\left(\lambda \frac{\partial T}{\partial x_{i}}\right)=C_{\mathrm{ef}}\left(\frac{\partial T}{\partial t}+v_{i} \frac{\partial T}{\partial x_{i}}\right)-Q_{V}
\end{aligned}
$$

where $\rho$ is a density $\left[\mathrm{kg} / \mathrm{m}^{3}\right], \boldsymbol{g}$ is acceleration of gravity $\left[\mathrm{m} / \mathrm{s}^{2}\right], \beta_{T}$ is a volume expansion coefficient due to heating $[1 / \mathrm{K}], T_{\text {ref }}$ is a reference temperature $[\mathrm{K}], \mu$ is a dynamic viscosity $[\mathrm{kg} /(\mathrm{m} \mathrm{s})]$, $K$ is porous medium permeability described by the Carman-Kozeny equation [4], $T=T\left(x_{i}, t\right)$ is temperature $[\mathrm{K}]$ at a point $x_{i}, v_{i}$ is a velocity vector $[\mathrm{m} / \mathrm{s}], \lambda=\lambda(T)$ is a thermal conductivity, 
$C_{\text {ef }}=C_{\text {ef }}(T)$ is an effective heat capacity with latent heat of fusion, evaporation taken into account [5].

Initial conditions and boundary conditions complete above governing equations [4].

\subsection{Heat sources}

The research is performed using Trumpf D70 laser head combined with TruDisk 12002 disk laser (Fig. 2).

Basic parameters of the laser beam are obtained using Prometec UFF100 beam analyzer, a diagnostic device for measuring high power lasers profile. The caustic obtained by the measurement is presented in Fig. 3.

Point kriging $[6,7]$ is used for a precise description of Yb:YAG laser power intensity distribution as a linear combination of observations in basic points. The estimate is a function of the weighted average

$$
\tilde{f}(x, y)=\sum_{i=1}^{n} w_{i} f\left(x_{i}, y_{i}\right),
$$

where $w_{i}$ are weight coefficients assigned to particular observations, $f\left(x_{i}, y_{i}\right)$ is the real value of the function (variable) at the measured point, $n$ is the number of sampling points that are considered in estimating of the variable within the circle of radius $r_{k}$ from estimated point. The distribution for focusing $z=0$ is presented in Fig. 4.

The volumetric heat source power distribution of a laser beam is calculated assuming the linear decrease of energy intensity with material penetration

$$
\begin{aligned}
& Q_{2}(x, y, z)=\eta Q_{S} \tilde{f}(x, y)\left(1-\frac{z}{s}\right), \\
& Q_{S}=\alpha P / \pi \omega_{0}^{2} s
\end{aligned}
$$

where $\eta$ is the absorption coefficient, $Q_{S}$ is the laser power per unit area $\left[\mathrm{W} / \mathrm{m}^{2}\right], P$ is pumped power $[\mathrm{W}], \omega_{0}$ is a radius of the beam $[\mathrm{m}], s$ is the heat source penetration depth $[\mathrm{m}], \alpha$ is a heat source coefficient (for the cone-like shape of the volume of a heat source the distribution $\alpha=3$ ).

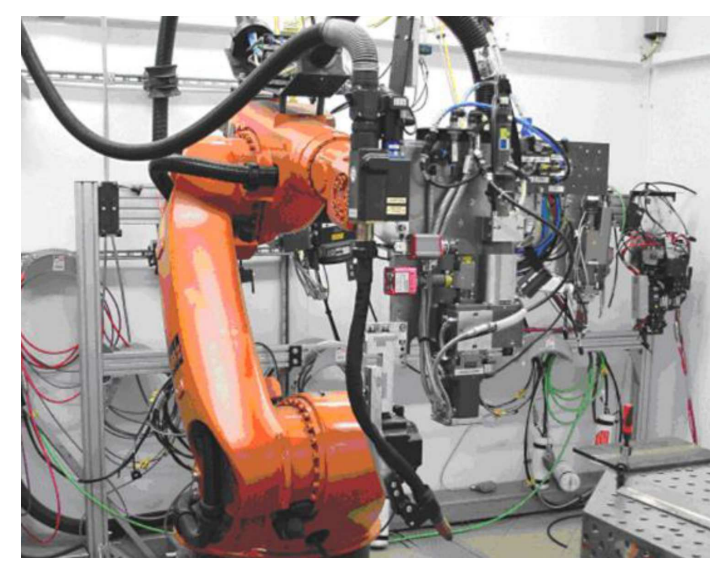

Fig. 2. Laser welding station.

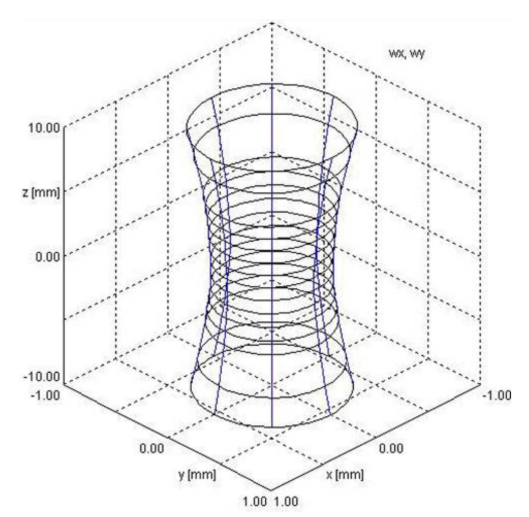

Fig. 3. Laser beam 3D caustic.
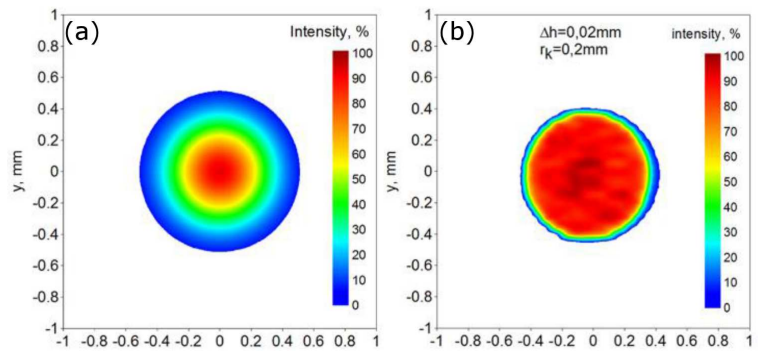

Fig. 4. Percentage distribution of laser power described by (a) Gaussian and (b) interpolated models.

Defined by Goldak [8] "double ellipsoidal" power distribution of the welding arc heat source is often used in modeling of arc welding process.

$$
Q_{1}=\left\{\begin{array}{l}
q_{1}(x, y, z)=\frac{6 \sqrt{3} f_{1} Q_{A}}{a b c_{1} \pi \sqrt{\pi}} \exp \left(-3 \frac{x^{2}}{c_{1}^{2}}\right) \\
\exp \left(-3 \frac{y^{2}}{a^{2}}\right) \exp \left(-3 \frac{z^{2}}{b^{2}}\right) \quad \text { for } x<x_{0}, \\
q_{2}(x, y, z)=\frac{6 \sqrt{3} f_{2} Q_{A}}{a b c_{2} \pi \sqrt{\pi}} \exp \left(-3 \frac{x^{2}}{c_{2}^{2}}\right) \\
\exp \left(-3 \frac{y^{2}}{a^{2}}\right) \exp \left(-3 \frac{z^{2}}{b^{2}}\right) \quad \text { for } x \geq x_{0},
\end{array}\right.
$$

where $a, b, c_{1}$, and $c_{2}$ are set of axes defining front ellipsoid and rear ellipsoid, $f_{1}$ and $f_{2}\left(f_{1}+f_{2}=2\right)$ represent distribution of the source energy at the front and the rear section of the source, thus resultant distribution of the source energy is total sum described as $Q_{1}(x, y, z)=q_{1}(x, y, z)+q_{2}(x, y, z)$ and $Q_{A}=\eta_{A} I U$ is the arc heat source power, where $I$ is a current intensity, $U$ is a voltage and $\eta_{A}$ is an efficiency of electric arc.

Arc + laser hybrid heat source distribution (Fig. 5) is considered as a product of "double ellipsoidal" Goldak's model and Yb:YAG interpolation model $\left(Q_{1}+Q_{2}\right)$.

\section{Results and discussion}

Computer simulation of hybrid welding processes was performed for sheets made of S355 steel in the system with leading laser beam heat source in the tandem. The dimensions of the sheets are $150 \times 40 \times 5 \mathrm{~mm}^{3}$. Analyzed domain was discretized by staggered grid with the spatial step set 


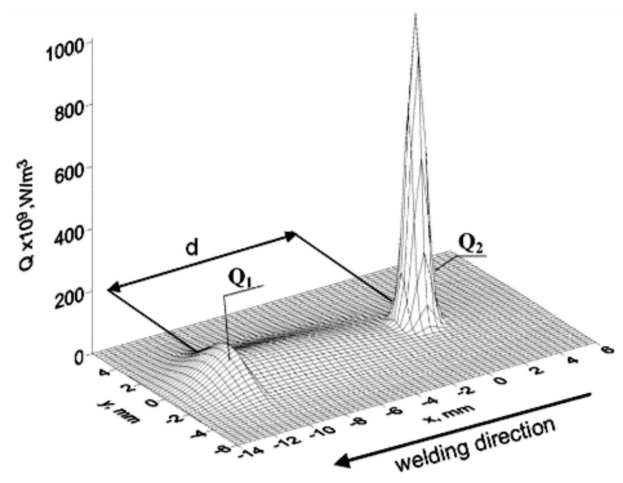

Fig. 5. Hybrid heat source power distribution at top surface $(z=0)$ of welded joint.

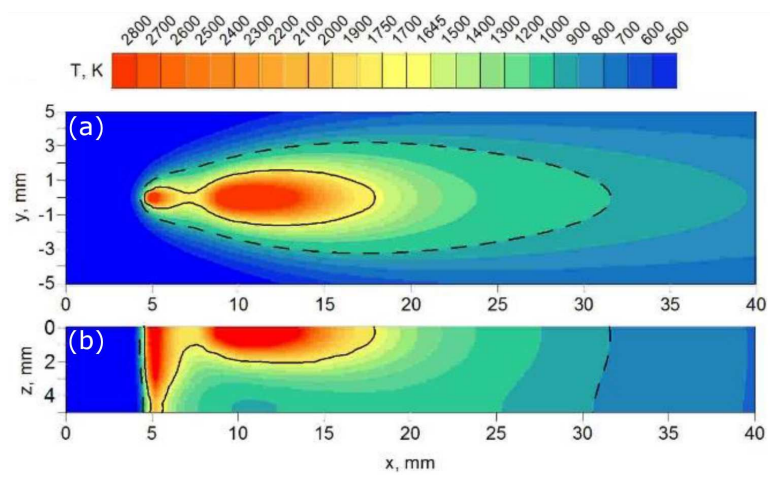

Fig. 6. Temperature distribution (a) at the top surface $(z=0)$ and (b) in longitudinal section $(y=0)$ of the joint. Laser beam focusing $z=0$.

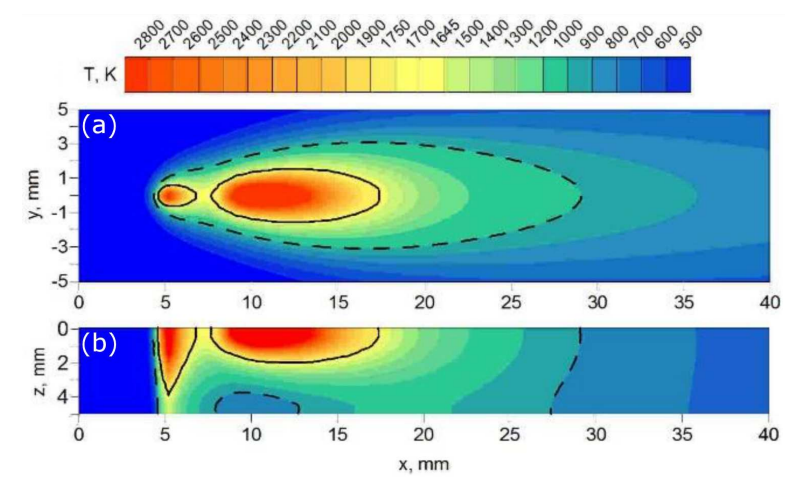

Fig. 7. As in Fig. 6, but for laser beam focusing $z=-5 \mathrm{~mm}$.

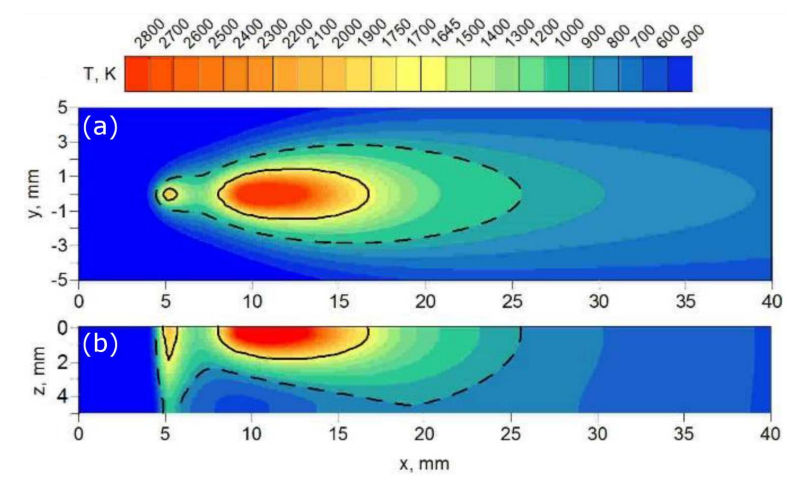

Fig. 8. As in Fig. 6, but for laser beam focusing $z=-10 \mathrm{~mm}$. to $0.1 \mathrm{~mm}$. Three simulations are performed with different laser beam focusing $z=0, z=-5 \mathrm{~mm}$ and $z=-10 \mathrm{~mm}$. Presented below (Figs. 6-8) temperature distributions show the differences of melted zone geometry (solid line) and heat affected zone geometry (dashed line) obtained in simulations.

\section{Conclusions}

The comparison of Figs. 6-8 shows significant differences in the metling zone at different focusing of Yb:YAG laser beam. In the case of focusing $z=0$, the synergy effect of the sources is visible (one welding pool is generated by the hybrid heat source).

For focusing $z=-5 \mathrm{~mm}$, the separation of heat sources activity zones is noticeable. The largest difference is present in the case of focusing with $z=-10 \mathrm{~mm}$. In the case of a defocused beam (Fig. 8) there is no full material penetration (less in the case of $z=-5 \mathrm{~mm}$ ).

The significant impact of the hybrid heat source power distribution on the temperature distribution and the welding pool as well as heat affected zones is noticeable. This distribution depends on the focusing of Yb:YAG laser beam (other technological parameters are the same).

Developed mathematical and numerical models can have a practical significance for technologists when selecting the proper technological parameters of the process, ensuring the desired quality of hybrid welded joints.

\section{References}

[1] P. Seyffarth, I.V. Krivtsun, Laser-Arc Processes and their Applications in Welding and Material Treatment, Taylor \& Francis, USA 2002.

[2] H.S. Kim, J.M. Yang, J. Korean Phys. Soc. 55, 1425 (2009).

[3] S.V. Patankar, Numerical Heat Transfer and Fluid Flow, Taylor \& Francis, USA 1990.

[4] M. Kubiak, W. Piekarska, Z. Saternus, T. Domański, Proced. Eng. 136, 88 (2016).

[5] M. Kubiak, W. Piekarska, Composit. Struct. 172, 29 (2016).

[6] S. Sakata, F. Ashida, M. Zako, Comput. Methods Appl. Mech. Eng. 192, 923 (2003).

[7] M. Kubiak, W. Piekarska, S. Stano, Int. J. Heat Mass Transf. 83, 679 (2015).

[8] J.A. Goldak, Computational Welding Mechanics, Springer, New York 2005. 